\title{
Are 128 Bits Long Keys Possible in Watermarking?
}

\author{
Patrick Bas ${ }^{1, \star}$ and Teddy Furon ${ }^{2}$ \\ 1 CNRS-LAGIS, Ecole Centrale de Lille, France \\ patrick.bas@ec-lille.fr \\ ${ }^{2}$ INRIA Research Centre Rennes Bretagne Atlantique, France \\ teddy.furon@inria.fr
}

The question raised in this poster is the following: is the key length of a watermarking system proportional to the key length of the seed used to generate the watermark? For example, if a watermark is generated from a binary sequence of size $n$, does it means that the key length is $2^{n}$ ?

As we shall see in this poster, the answer is no! We will show how the keylength in Watermarking strongly relies on

(1) the robustness of the watermarking scheme,

(2) the embedding and decoding functions,

(3) the observations available to the adversary.

The goal of this poster is to propose techniques to practically compute the keylength of a watermarking scheme. To do so we first compute the probability $p$ that the adversary has access to the watermarking channel by picking a random key. This probability can be computed using three mathematical subsets: the embedding region, the decoding region and the region of equivalent keys, the latter being defined w.r.t both the embedding and decoding region. With this formulation, $p$ is the probability that a random key belongs to the region of equivalent keys and the effective key length is given by

$$
\ell=-\log _{2} p .
$$

We will illustrate in the poster how to practically compute $\ell$ on various popular watermarked schemes (Spread Spectrum, Improved Spread Spectrum, Distortion Compensated Quantization Index Modulation, Normalized Correlation) using different means such as mathematical derivations, Monte-Carlo simulations or geometrical estimation, and under different scenarios such as without any observation or taking into account a set of watermarked contents.

More informations about this work on http://arxiv.org/abs/1202.3562

\footnotetext{
^ P. Bas' work was partly founded by the French National Research Agency program referenced ANR-10-CORD-019 under the Estampille project. 